\title{
ROLE OF GUT MICROBIOTA MODULATION IN HEALTH AND PRODUCTION OF PIGS
}

\author{
A. K. DAS ${ }^{1 *}$, N. R. CHOWDHURY ${ }^{2}$, P. K. NANDA ${ }^{1}$, P. DANDAPAT ${ }^{1}$, S. BATABYAL $^{2}$, \\ S. BISWAS ${ }^{3}$ AND P. K. DAS ${ }^{4}$
}

${ }^{1}$ Eastern Regional Station, ICAR-Indian Veterinary Research Institute, Kolkata-700 037, West Bengal, India

${ }^{2}$ Department of Veterinary Biochemistry, West Bengal University of Animal and Fishery Sciences, Kolkata-700 037, West Bengal, India

${ }^{3}$ Department of Livestock Products Technology, West Bengal University of Animal and Fishery Sciences, Kolkata-700 037, West Bengal, India

${ }^{4}$ Department of Veterinary Physiology, West Bengal University of Animal and Fishery Sciences, Kolkata-700 037, West Bengal, India

\begin{abstract}
The importance of pig as a livestock species contributing to the meat production is well known. However, in recent years, the gut microbiome of pig has received considerable attention, as pigs serve as excellent biomedical models for human health compared to other non-primates. The pig gastro-intestinal tract harbours a lot of microbes, including bacteria, fungi, protozoa, viruses and bacteriophages. These microbes form a complex symbiotic ecosystem with the host and play pivotal role in maintaining various physiological, nutritional and immunological mechanisms of pigs. However, factors including rearing and management, environmental and antimicrobials used affect the microbial population in the pig gut affecting their health and growth. So modulation of gut microbiota is required which not only help in improving the health of pigs leading to higher production but also generate more revenue for the pig industry. In recent days, extensive studies have been made on gut microbiota using probiotics along with other prebiotic and feeding highly fermentable carbohydrates and microbial transplantation to reduce anti-microbial dependence for growth, maintenance of pig-health in order to elevate production. The review highlights various factors affecting pig gut microbiota and their role in health maintenance leading to efficient production of pigs.
\end{abstract}

Key words: Gut microbiota, Health, Modulation, Pig, Production

\section{Introduction}

The pig gastro-intestinal tract harbours a lot of microbes, including bacteria, fungi, protozoa, viruses, and bacteriophages. Out of approximately $10^{10}-10^{14}$ cells, more than $98 \%$ of isolated genetic sequences present in the gut come from bacteria (Turner, 2018). These microbes form a complex symbiotic ecosystem with the host and play pivotal role in maintaining various physiological, nutritional and immunological mechanisms of the pigs (Lee and Mazmanian, 2010; Brestoff and Artis, 2013). These mechanisms help in maintenance of health and also uphold production aspects of the pigs in farms. Besides harbouring beneficial microbes, the gut microbiome also consists of various pathogenic bacteria like Escherichia coli, Salmonella, Clostridium etc. which are regarded as opportunistic pathogens. 
Any sort of shift towards abnormality causes pathogenicity exerted by those pathogens in the gut accounting for morbidity and mortality among the pig population. So, to combat that, antimicrobials are routinely being used as a prophylactic and health-managemental procedure to minimize the loss in animal husbandry sector and livestock industries. Besides, the antimicrobials are used during weaning of piglets and also as growth factors to meet the production goals in the industry. This not only results into deteriorative effects on the gut microbiome of the weaned piglets, but also disrupt the gut lumen environment leading to post-weaning diarrhoea (Lallès et al., 2004; Konstantinov et al., 2006). Excessive usages of the antimicrobials hamper the beneficial microbes and may cause hindrance in obtaining proper health of the pigs. Growth promoting antimicrobials also elevate the population of antimicrobial resistant bacteria which is a major health concern (Dibner and Richards, 2005). Extensive studies on gut microbiota, using probiotics along with other prebiotic and feeding highly fermentable carbohydrates and microbial transplantation, are the way-outs to reduce anti-microbial dependence for growth and maintenance of pig-health in order to elevate production. This review highlights various factors affecting pig gut microbiota and their role in health maintenance leading to efficient production of pigs.

\section{Intestinal microbiota of pig}

Pig gut microbiome is comprised of numerous bacterial and non-bacterial components. The population of these components depends not only on age, but also their feeding habits, rearing environment, management types etc.

Bacterial components of the microbiome: The microbial colonization in gut of the piglets starts just after the birth (Fouhse et al., 2016). Initial colonization is made with Escherichia coli and
Streptococcus spp., yielding an anaerobic environment to help Bacteroides spp., Bifidobacterium spp., Clostridium spp., Lactobacillus spp. to colonize (Konstantinov et al., 2006; Petri et al., 2010). Among them, Lactobacilli are the major bacteria in the piglet gut during the pre-weaning phase (Konstantinov et al., 2006; Petri et al., 2010)

In the post weaning phase, a study by Kim et al. (2011) classified the bacteria found in the pig-faeces into five phyla: Firmicutes, Bacteroidetes, Proteobacteria, Actino-bacteria and Spirochaetes. Among them, the largest proportion is made by the phylum Firmicutes, followed by Bacteroidetes, together accounting for $90 \%$ of all the bacterial population primarily at the 3 weeks aged pigs. Along with the time, the Firmicutes population increase with respect to that of the phylum Bacteroidetes. Surprisingly, non-classified groups of bacteria also increase their population. Prevotella spp. are the most predominant bacteria accounting for $30 \%$ of all classifiable bacteria when the pigs are aged 10 weeks, but, their concentration decreased to $3.5-4 \%$ only at the age of 22 weeks (Kim et al., 2011). The most abundant species are: Prevotella spp., Anaerobacter spp., Streptococcus spp., Lactobacillus spp., Coprococcus spp., Megasphaera spp., Subdoligranulum spp., Blautia spp., Oscillibacter spp., Faecalibacterium spp., Dialister spp., Sarcina spp., Butyricoccus spp. (Table 1).

Non-bacterial components of the microbiome: In a bid to find the non-bacterial components in pig gut, Urubschurov et al. (2011) screened pig faeces using 26S rRNA gene coupled with denaturing gradient gel electro-phoresis (DGGE) and DNA sequencing. The researchers isolated yeast, such as Kazachtania slooffiae, Galactomyces geotrichum, Candida catenulate and C. glabrata using cultural technique 
Table 1. Major bacterial population in different intestinal portions

\begin{tabular}{cl}
\hline Portion of the Intestine & \multicolumn{1}{c}{ Major bacterial species/phylum } \\
\hline Jejunum & Firmicutes, Proteobacteria, Cyanobacteria, Actinobacteria \\
Ilium & Firmicutes, Proteobacteria \\
Colon and caecum & Firmicutes, Bacteroidetes \\
\hline
\end{tabular}

Adapted from Isaacson et al. (2011)

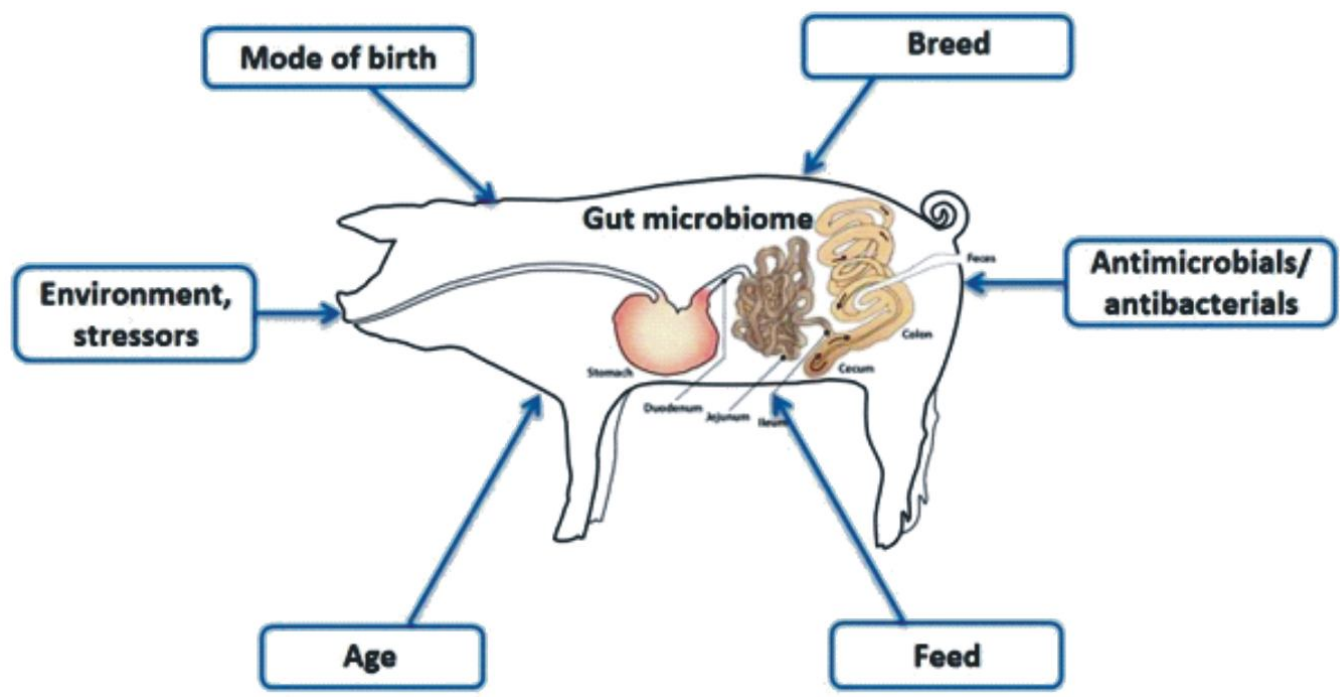

Fig. 1. Various factors affecting gut microbiome of pigs (Picture-Holman et al., 2017)

(Urubschurov et al., 2011). In a separate study, Shan et al. (2011) showed that the pig faeces contained $(99 \%)$ RNA viruses from the families: Picornaviridae, Astroviridae, Coronaviridae and Caliciviridae. The DNA viruses present are of the Circoviridae and Parvoviridae families.

\section{Factors affecting the gut microbiota in pigs}

Several factors potentially affect the microbial population in the pig gut affecting the health and growth of the pigs, starting from the breed of the pig, management practices adopted, effect of host genetics and environmental factors (e.g., pen, kinship), age, body weight, obesity, diet, types of enrichment and antimicrobials used (Turner, 2018; CrespoPiazuelo et al., 2019; Wang et al., 2019)

Birth: Studies have shown that the pig microbial community is primarily influenced by the microbial encounters happened in the early stages of life i.e. the act of birth (Patil et al., 2020). In fact, porcine gut microbial composition is heavily dependent upon the mode of the delivery (vaginal/normal delivery or caesarean section) along with the nutrition provided to the piglets (Patil et al., 2020). It has also been reported that maternal transfer of gut microbial population is possible during parturition via umbilical cord (Patil et al., 2020). Pigs born via normal mode of parturition have higher gut bacterial density and higher concentrations of acetate, propionate and butyrate, in comparison with the pigs born via caesarean section (Wang et al., 2013).

Breed: Breed of the pig may also affect gut microbiota composition. On examining the faecal microbial community, Bian et al. (2016) 
reported a different bacterial community structure in breeds of Meishan (a Chinese domestic obese breed) and Yorkshire (lean-type breed) piglets on day 14 and day 49. The Meishan sows had relatively higher abundances of Firmicutes but lower Bacteroidetes than Yorkshire sows, this may potentially affect postnatal development in piglets. Further, the authors ( $\mathrm{Mu}$ et al.) concluded that Prevotella, a Gram-negative bacterium, was less abundant in Meishan breed compared to Yorkshire piglets (Mu et al., 2019). In studying the gut microbiota profile of different pig breeds viz. Tibetan pig (TP), Yorkshire pig (YP) and Rongchang pig (RP) based on high-through put pyrosequencing; Diao et al. (2016) opined that YP exhibited a lower Firmicutes/Bacteroidetes ratio whereas TP had higher levels of bacteria from Spirochaetes compared to other breeds. More diverse gut microbiome composition has been reported in Landrace pigs compared to the other two breeds, Duroc and Large WhiteYorkshire (LWY) (Bergamaschi et al., 2020).

In general, two major types of bacteria, Firmicutes and Bacteroidetes found in the pig gut and faecal materials, are also present in different proportions in different pig breeds (Patil et al., 2020). Like, in Chinese Jinhua pigs, $70.4 \%$ faecal bacterial population is of the Firmicutes while the Bacteroidetes comprises $14.4 \%$ (Yang et al., 2018a). In western breeds, the scenario changes quite drastically (Table 2).

Age: Microbial colonisation within the pig gut is also influenced by age of the pigs too (Zhao et al., 2015; Holman et al., 2017). The earliest colonisers (between birth and 2 days of age) are Escherichia sp., Clostridium sp., Fusobacterium sp., Streptococcus sp. and Enterococcus sp. (Dowarah et al., 2017). About $34 \%$ of the total microbial population of the gut is seen to be comprised of Clostridium spp. at the 6 hours age, which reduces to $1 \%$ when the pigs become 20 days (Petri et al., 2010). Bacteria from the family Enterobacteriaceae are not found during those early days (Petri et al., 2010). In the first 5 days after birth, strict aerobic and facultative anaerobic bacteria are seen which are replaced by the strict anaerobic bacteria gradually by the age of 22 days (Inoue et al., 2005).

Likewise, analysing the gut microbiomes of newborns to 10 years old micro-pigs, Lim et al. (2019) found sequential changes of the gut microbiome in relation to age. With an increase in age, Bacteroides increased whereas beneficial microbes, including probiotic bacteria and short chain fatty acids (SCFA) producers, were found more in younger pigs (Lim et al., 2019).

In a study by Mach et al. (2015), the composition of faecal microbiomes of healthy piglets at 14 , 36, 48, 60 and 70 days after birth together assessed by $16 \mathrm{~S}$ rRNA gene 454-pyrosequencing revealed Bacteroides, Oscillibacter, Escherichial Shigella, Lactobacillus and unclassified Ruminococcaceae genera as the microbiota of suckling piglets. On the other hand, Acetivibrio, Dialister, Oribacterium, Succinivibrio and Prevotella genera were more dominant in piglets after weaning (Mach et al., 2015).

While studying the gut microbial community compositions in different age groups (from 25

Table 2. Western pig breeds and their major gut-bacterial composition

\begin{tabular}{ccc}
\hline Western pig breed & Firmicutes present $\mathbf{( \% )}$ & Bacteroidetes present (\%) \\
\hline Yorkshire & 42.0 & 51.4 \\
Duroc & 39.6 & 57.0 \\
Landrace & 45.6 & 47.6
\end{tabular}

Adopted from Pajarillo et al. ( 2014, 2015) 
to 240 days of age), Ke et al. (2019) found that the gut microbiota of pre-weaning piglets was predominated by two enterotypes, Fusobacterium spp. and p-75-a5, respectively whereas Prevotella spp. and Treponema spp. were the main enterotypes at later ( $>80$ days).

Diet and feed efficiency: Diet has significant impact on the gut microbial population and a proper and balanced diet to pig at different stages of growth also helps in preventing dysbiosis (Doré and Blottière, 2015), the reason behind numerous chronic diseases. On the other hand, any dietary changes or unbalanced rations coupled with low feed digestibility can change the richness of beneficial bacterial populations in favour of pathogenic bacteria in the gut, which may hamper the animal health and productivity.

Feed efficiency being an important economic and environmental parameter that is wellstudied to find out the feasibility of the farm and swine industry is no exception to this. In fact, the gut microbiota plays an important role in carbohydrate metabolism and helps in nutrient uptake after metabolizing various food components, particularly indigestible polysaccharides (Yang et al., 2017). Since the gut microbiota plays a pivotal role in nutrient digestibility, the differences in gut microbiome composition may impact feed efficiency among the swine breeds (Bergamaschi et al., 2020), thereby affecting its production causing considerable economic loss to the sector.

Recent studies have shown that Ruminococcus spp. and Lactobacillus spp. lower the feed intake of pigs while the Prevotella spp. show just the opposite (Yang et al., 2018b), justifying the role of Prevotella spp. as the keystone for appetite control of the pigs. When the pigs shift from milk-based diet to solid feed diet (during weaning), bacterial composition within the gut also changes (De Rodas et al., 2018). Even paternal (Duroc-DR) and maternal (LandraceLR and Large White-LW) lines have significant effect on feed efficiency and gut microbiome composition. In a study, it was found that the microbial communities in DR breed had better proportion of the Catenibacterium and Clostridium genera than the other two breeds whereas LR pigs had significantly higher representation of Bacteroides than LW (Bergamaschi et al., 2020).

Different researchers have even identified the relationship between gut microbiota diversity and feed efficiency in pigs. While the bacterial species belonging to the genera Bacteroides, Cellulosilyticum, and Prevotella are reported to be more abundant in pigs with low feed efficiency, Oscillibacter and Rhodococcus are described in feed efficient animals (McCormack et al., 2017; Tan et al., 2017).

Studying the gut microbiota of pigs from birth to market, i.e. lactation, nursery, growing and finishing, Li et al. (2020) found a dramatic change in gut microbiome structure during weaning, when solid food replaced sow milk. Even when on solid diet during different stages of growth, the change was gradual indicating the role of diet in influencing the composition of gut microbiota. A diet of weaning piglets supplemented with a cocktail of feed additives (cranberry extract, encapsulated carvacrol, yeast-derived products, and extra vitamins A, $\mathrm{D}, \mathrm{E}$, and $\mathrm{B}$ complex) and bovine colostrum has also been reported to modulate intestinal microbiota and improved the growth performance of piglets (Lo Verso et al., 2020).

Dietary fibre: Dietary fibres are plant polysaccharides that are potentially available for bacterial fermentation in the large intestines of single-stomached animals (Bindelle et al., 2008). The dietary fibre component of feed is reported to be beneficial for intestinal health and animal welfare, as it decreases the diarrhea 


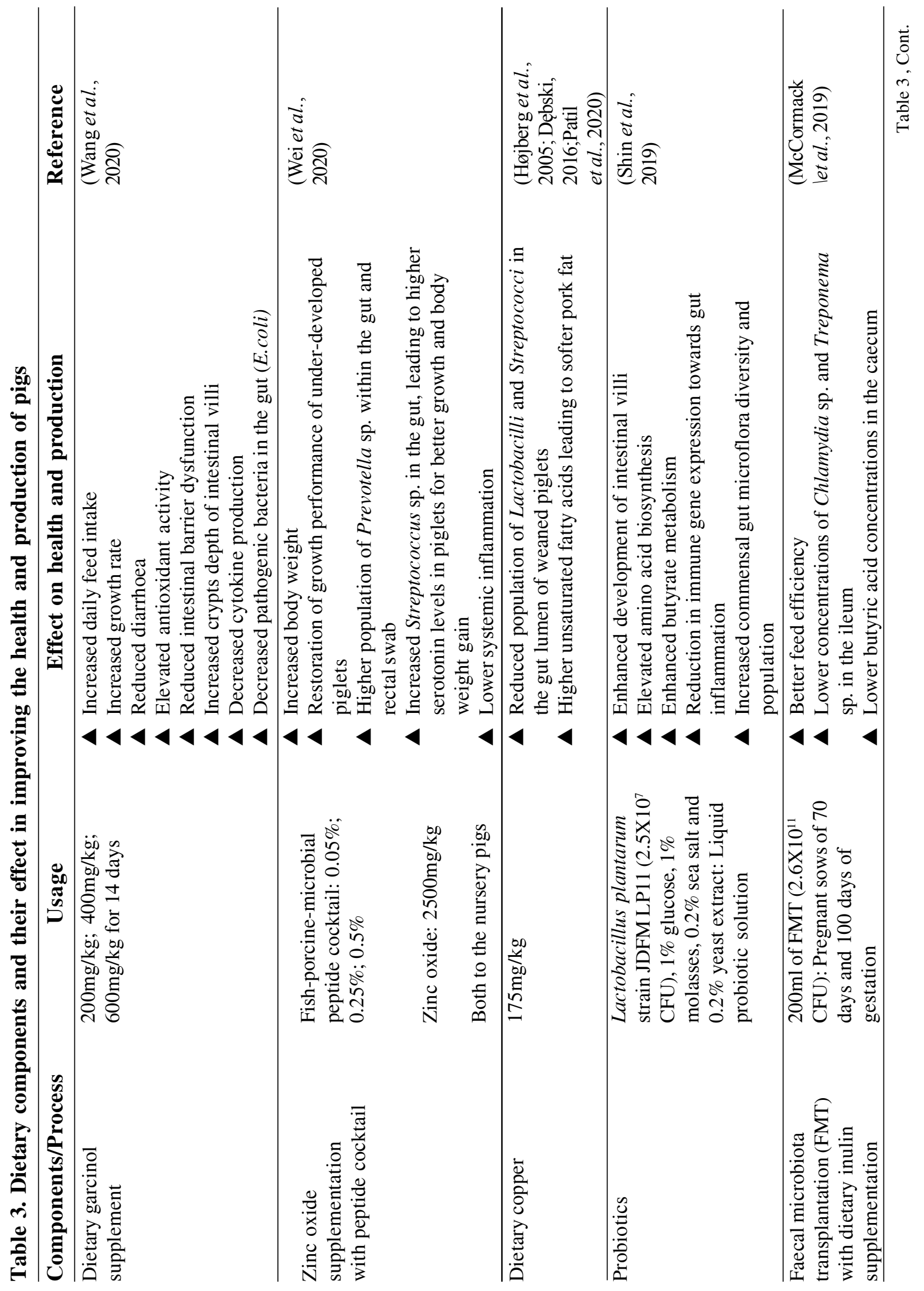


Gut microbiota and pig production

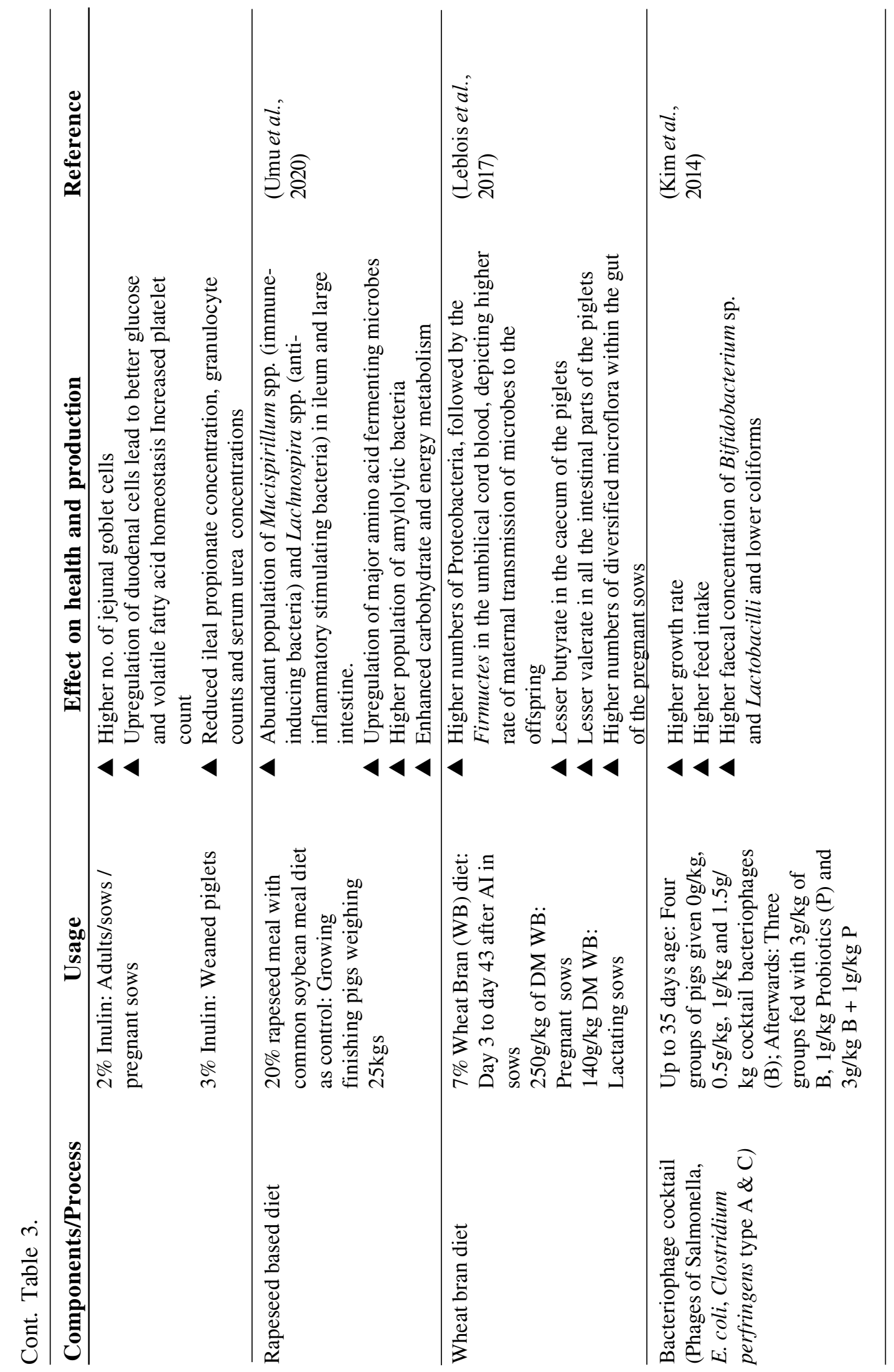


incidence in pigs around the time of weaning. In fact, upon bacterial fermentation of the dietary fibres, short chain organic acids (acetate, lactate, propionate and butyrate) get formed, which help in digestive tract development by potentiating the gut epithelia (Montagne et al., 2003). These acids also inhibit growth of Salmonella, E. coli and Clostridium (Pickard et al., 2017). The non-starch polysaccharides stimulate the growth of commensals within the gut, increase the short chain organic acid production, lower the colon $\mathrm{pH}$ and increase the length of the intestinal villi (Bach Knudsen et al., 2012; Lindberg, 2014). Some studies also suggest that the low fat and high fibre diet fed pigs have more numbers of Lactobacillus, Bifidobacterium and Faecalibacterium prausnitzii population within the gut whereas, the high fat and low fibre diet fed pigs contained more population of the bacteria under Enterobacteriaceae family (Heinritz et al., 2016). The addition of guar gum or cellulose to a standard diet has also been reported to increase the ileal Bifidobacteria and Enterobacteria populations in growing pigs (Owusu-Asiedu et al., 2006).

The levels of proteins or fibers and their source also influence the diversity and composition of the gut microbiota, particularly of weaning piglets (Rist et al., 2013; Pieper et al., 2015). For example, in weaning pigs, pectin enriched diets as well as a soybean meal is reported to decrease the relative abundance of Lactobacillus and increase Prevotella sp. in the colon (Tian et al., 2017). On the other hand, a protein source from fish is often linked to an increase in Escherichia/Shigella group (Cao et al., 2016).

Dietary copper: Copper is an essential micronutrient and if used in feed offer many biological functions in animals. It protects the animal from oxidative stress, helps in iron metabolism, improves the activity of digestive enzymes, to name a few (Zhang et al., 2019). Copper in feed of weaned piglets is reported to improve body weight gain and feed conversion ratio and reduce the frequency of diarrhoea. Therefore, copper supplementation with the feed is a tradition that pig industry practices due to its anti-microbial and growth promoting properties (Patil et al., 2020). This practice helps to reduce the colonies of Lactobacillus and Streptococcus in the pig gut (Højberg et al., 2005). High amounts of dietary copper increase the content of unsaturated fatty acids leading to production of softer pork fat (Dêbski, 2016).

Probiotics and prebiotics: Probiotics are a group of micro-organisms, when administered in adequate amounts, the individual gets overall health improvement (Linares et al., 2017). Probiotics improve nutrient digestion and metabolism, resulting into a better growth of animals and thus the production performance of the animal gets improved markedly (Wang and Donovan, 2015). Probiotic supplementation increases beneficial bacteria within the pig gut (like Lactobacilli) and decrease the population of harmful bacteria (like E. coli, Clostridia spp. etc.) (Galdeano et al., 2010) leading to much higher growth rate and body weight gain, meeting the production target in the pig industries (Wells et al., 2005).

Prebiotics are the compounds which help to upregulate the population of beneficial microorganisms in the gut (Hutkins et al., 2016). Prebiotics potentiate the works of probiotics also, leading to additive effect on that of the probiotics to help elevate growth rate of pigs, meeting the production target in the pig industry (ÇEtin et al., 2005; Yin et al., 2008). Also, prebiotic supplementation elevates interleukin production in the pig's body (ÇEtin et al., 2005; Yin et al., 2008). Prebiotics, such as lactulose, provided to the weaned piglets showed marked improvement in IgG, IgM and IgA production upon challenging with 
Salmonella enterica subspecies enterica serovar Typhimurium (Pieì et al., 2007).

Antibacterials: Antibacterials like penicillins, tylosin, sulfamethazine, tetracyclines are often used as growth promoters in the pig industry (Allen et al., 2011; Kim et al., 2012, 2016; Looft et al., 2014). But these agents have negative impact on the commensals living in the pig gut (Dibner and Richards, 2005). Excessive usage of antimicrobials cause to increase immune-tolerance, leading to insufficient immune response against the antimicrobial-resistant pathogenic species infections in future (Schokker et al., 2014). In recent days, the negative effects of antibacterials on gut microbiota and host health have been recognized. Further, long term uses of antibacterial not only decrease bacterial diversity and their relative abundance but also develop antibiotic resistance. So to minimise the effects of antibiotics on the intestinal microbiota, tailored antibiotic treatment is often advocated to avoid this collateral damage.

Stressors and weaning: It is well established that any form of stress like weaning, heat stress, etc affects the growth, reproduction, productivtiy and diseases susceptability in livestock (Parkunan et al., 2015). There are six phases in a piglet's life and weaning is considered as the critical phase among all. The stress caused as a result of weaning not only changes microbial composition and function but also alters the microbial metabolic profiles in the intestine (Li et al., 2018). During this period, functional changes in the gut microbiome is observed when the host animal undergoes stressful conditions like confinements, weaning, heat etc. (Patil et al., 2020). Due to the stress exerted because of weaning, the ileal Lactobacilli population undergoes marked changes, hampering the production criteria for pigs (Janczyk et al.,
2007). Post weaning periods depict uphold of Bacteroidetes population over Firmicutes in the pig gut (Pajarillo et al., 2014).

\section{Conclusion}

The importance of pig as a livestock species contributing to the meat production is well known. However, in recent years, the gut microbiome of pig has received considerable attention, as pigs serve as excellent biomedical models for human health compared to other non-primates. Pigs have a relatively high degree of similarity index with humans in terms of the genome, protein sequences as well as immune responses. So swine as a model is more preferred over other species in studying the interaction of early gut microbiota and its impacts on health risks. With the development of modern analytical methods such as fluorescent in situ hybridization, temperature gradient gel electrophoresis, DGGE, molecular tools, particularly next generation sequencing, it is now possible to identify and analyse the composition of microbial communities of gut at different stages of growth. This helps in better understanding the gut microbial composition which could be used as a biomarker to predict health status of piglets, contributing better immunological protection to the host and thus, lesser diseases will occur. As transition through weaning is a crucial period in pig farm management, gut microbe modulation comes out handy. This not only elevates the population of the microbes within the gut, but also decreases the harmful, pathogenic microbes, so that the beneficial commensals get the upperhand within the gut. So modulation of gut microbe not only helps in improving the health of the pigs leading to higher production but also generate more revenue for the pig industry. Formulating a commercial diet during this transition period is important for optimum gut health of pigs. As the intestinal microbiome is significantly affected by dietary changes (from milk to solid food), more research is needed 
in this front to develop feed with nonantimicrobial alternatives such as pre- and probiotics, essential oils, enzymes, macro- and micronutrients etc. as additives to restore intestinal balance and overcome crucial weaning transition. This will not only ensure optimum composition of the gastrointestinal microflora in piglets by maintaining a balance

\section{REFERENCES}

Allen HK, Looft T, Bayles DO, Humphrey S, Levine UY et al., 2011. Antibiotics in feed induce prophages in swine fecal microbiomes. mBio, 2(6): e0026011, doi: 10.1128/mBio.00260-11

Bach Knudsen KE, Hedemann MS and Lærke HN, 2012. The role of carbohydrates in intestinal health of pigs. Anim Feed Sci Technol, 173(12): 41-53, doi: 10.1016/j.anifeedsci. 2011.12.020

Bergamaschi M, Tiezzi F, Howard J, Huang YJ, Gray KA et al., 2020. Gut microbiome composition differences among breeds impact feed efficiency in swine. Microbiome, 8: 110, doi: 10.1186/ s40168-020-00888-9

Bian G, Ma S, Zhu Z, Su Y, Zoetendal EG et al., 2016. Age, introduction of solid feed and weaning are more important determinants of gut bacterial succession in piglets than breed and nursing mother as revealed by a reciprocal cross-fostering model. Environ Microbiol, 18(5): 1566-1577, doi: $10.1111 / 1462-2920.13272$

Bindelle J, Buldgen A and Leterme P, 2008. Nutritional and environmental consequences of dietary fibre in pig nutrition: A review. Biotechnol Agron Soc Environ, 12(1): 3247-3256

Brestoff JR and Artis D, 2013. Commensal bacteria at the interface of host metabolism and the immune system. Nat Immunol, 14(7): 676-684, doi: 10.1038/ni.2640

Cao KF, Zhang HH, Han HH, Song Y, Bai XL et al., 2016, Effect of dietary protein sources on the small intestine microbiome of weaned piglets based on high-throughput sequencing. Lett Appl Microbiol, 62(5): 392-398, doi: 10.1111/ lam. 12559

ÇEtin N, Güçlü BK and ÇEtin E, 2005. The effects of between beneficial and potentially pathogenic bacteria but also help the industry to get rid of the antibacterial/antimicrobial dependence to combat diseases or to promote growth. Further, research is also needed in understanding the impact of routine farm practices and farm environment having any role on a piglet's microbiome.

probiotic and mannanoligosaccharide on some haematological and immunological parameters in turkeys. J Vet Med Ser A, 52(6): 263-267, doi: 10.1111/j.1439-0442.2005.00736.x

Crespo-Piazuelo D, Migura-Garcia L, Estellé J, CriadoMesas L, Revilla M et al., 2019. Association between the pig genome and its gut microbiota composition. Sci Rep, 9: 8791, doi: 10.1038/ s41598-019-45066-6

De Rodas B, Youmans BP, Danzeisen JL, Tran H and Johnson J, 2018. Microbiome profiling of commercial pigs from farrow to finish. J Anim Sci, 96(5): 1778-1794, doi: 10.1093/jas/sky 109

Dêbski B, 2016. Supplementation of pigs diet with zinc and copper as alternative to conventional antimicrobials. Pol J Vet Sci, 19(4): 917-924, doi: 10.1515/pjvs-2016-0113

Diao H, Yan HL, Xiao Y, Yu B, Yu J et al., 2016. Intestinal microbiota could transfer host gut characteristics from pigs to mice. BMC Microbiol, 16: 1-16, doi: 10.1186/s12866-016-0851-z

Dibner JJ and Richards JD, 2005. Antibiotic growth promoters in agriculture: history and mode of action. Poult Sci, 84(4): 634-643, doi: 10.1093/ ps/84.4.634

Doré $\mathrm{J}$ and Blottière $\mathrm{H}, 2015$. The influence of diet on the gut microbiota and its consequences for health. Curr Opin Biotechnol, 32: 195-199, doi: 10.1016/j.copbio.2015.01.002

Dowarah R, Verma AK, Agarwal N, Patel BHM and Singh P, 2017. Effect of swine based probiotic on performance, diarrhoea scores, intestinal microbiota and gut health of grower-finisher crossbred pigs. Livest Sci, 195: 74-79, doi: 10.1016/j.livsci.2016.11.006 
Fouhse JM, Zijlstra RT and Willing BP, 2016. The role of gut microbiota in the health and disease of pigs. Anim Front, 6(3): 30-36, doi: 10.2527/ af.2016-0031

Galdeano CM, de Moreno de Leblanc A, Dogi C and Perdigón G, 2010. Lactic Acid Bacteria as Immunomodulators of the Gut-Associated Immune System. In: Biotechnology of Lactic Acid Bacteria: Novel Applications eds., WileyBlackwell, Oxford, UK, pp124-140

Heinritz SN, Weiss E, Eklund M, Aumiller T, Louis $\mathrm{S}$ et al., 2016. Intestinal microbiota and microbial metabolites are changed in a pig model fed a high-fat/low-fiber or a low-fat/high-fiber diet. PLoS One, 11: e0154329, doi: 10.1371/ journal.pone.0154329

Højberg O, Canibe N, Poulsen HD, Hedemann MS and Jensen BB, 2005. Influence of dietary zinc oxide and copper sulfate on the gastrointestinal ecosystem in newly weaned piglets. Appl Environ Microbiol, 71(5): 2267-2277, doi: 10.1128/AEM.71.5.2267-2277.2005

Holman DB, Brunelle BW, Trachsel J and Allen HK, 2017. Meta-analysis to define a core microbiota in the swine gut. mSystems, 2(3): e00004-17, doi: 10.1128/mSystems.00004-17

Hutkins RW, Krumbeck JA, Bindels LB, Cani PD, Fahey Jr. G et al., 2016. Prebiotics: why definitions matter. Curr Opin Biotechnol, 37: 1-7, doi: 10.1016/j.copbio.2015.09.001

Inoue R, Tsukahara T, Nakanishi N and Ushida K, 2005. Development of the intestinal microbiota in the piglet. J Gen Appl Microbiol, 51(4): 257-265, doi: 10.2323 /jgam.51.257

Isaacson R, Borewicz K, Kim HB, Vannucci F, Gebhart $\mathrm{C}$ et al., 2011. Lawsonia intracellularis increases Salmonella enterica levels in the intestines of pigs. In: Conference of Research Workers in Animal Diseases. Chicago, Illinois, pp107

Janczy KP, Pieper R, Smidt H and Souffrant WB, 2007. Changes in the diversity of pig ileal Lactobacilli around weaning determined by means of $16 \mathrm{~S}$ rRNA gene amplification and denaturing gradient gel electrophoresis. FEMS Microbiol Ecol, 61(1): 132-140, doi: 10.1111/j.15746941.2007.00317.x
Ke S, Fang S, He M, Huang X, Yang H et al., 2019. Age-based dynamic changes of phylogenetic composition and interaction networks of health pig gut microbiome feeding in a uniformed condition. BMC Vet Res, 15: 172, doi: 10.1186/ s12917-019-1918-5

Kim HB, Borewicz K, White BA, Singer RS, Sreevatsan $\mathrm{S}$ et al., 2011. Longitudinal investigation of the age-related bacterial diversity in the feces of commercial pigs. Vet Microbiol, 153(1-2): 124133, doi: 10.1016/j.vetmic.2011.05.021

Kim HB, Borewicz K, White BA, Singer RS, Sreevatsan $\mathrm{S}$ et al., 2012. Microbial shifts in the swine distal gut in response to the treatment with antimicrobial growth promoter, tylosin. Proc Natl Acad Sci USA, 109(38): 15485-15490, doi: 10.1073/pnas.1205147109

Kim J, Guevarra RB, Nguyen SG, Lee JH, Jeong DK et al., 2016. Effects of the antibiotics growth promoter tylosin on swine gut microbiota. J Microbiol Biotechnol, 26(5): 876-882, doi: 10.4014/jmb. 1512.12004

Kim KH, Ingale SL, Kim JS, Lee SH, Kwon IK et al., 2014. Bacteriophage and probiotics both enhance the performance of growing pigs but bacteriophage are more effective. Anim Feed Sci Technol, 196: 88-95, doi: 10.1016/ j.anifeedsci.2014.06.012

Konstantinov SR, Awati AA, Williams BA, Miller BG, Jones P et al., 2006. Post-natal development of the porcine microbiota composition and activities. Environ Microbiol, 8(7): 1191-1199, doi: $10.1111 /$ j.1462-2920.2006.01009.x

Lallès J-P, Boudry G, Favier C, Le Floc'h N, Luron I et al., 2004. Gut function and dysfunction in young pigs: physiology. Anim Res, 53(4): 301-316, doi: 10.1051/animres:2004018

Leblois J, Massart S, Li B, Wavreille J, Bindelle J et al., 2017. Modulation of piglets' microbiota: differential effects by a high wheat bran maternal diet during gestation and lactation. Sci Rep, 7: 1-11, doi: 10.1038/s41598-017-07228-2

Lee YK and Mazmanian SK, 2010. Has the microbiota played a critical role in the evolution of the adaptive immune system? Science, 330(6012): 1768-1773, doi: 10.1126/science. 1195568 
Li Y, Guo Y, Wen Z, Jiang X, Ma X et al., 2018. Weaning stress perturbs gut microbiome and its metabolic profile in piglets. Sci Rep, 8: 18068, doi: $10.1038 /$ s41598-018-33649-8

Li Y, Wang X, Wang XQ, Wang J and Zhao J, 2020. Life-long dynamics of the swine gut microbiome and their implications in probiotics development and food safety. Gut Microbes, 11(6): 1824-1832, doi: 10.1080/19490976.2020. 1773748

Lim MY, Song EJ, Kang KS and Nam Y-D, 2019. Agerelated compositional and functional changes in micro-pig gut microbiome. GeroScience, 41: 935-944, doi: 10.1007/s11357-019-00121-y

Linares DM, Gómez C, Renes E, Fresno JM, Tornadijo ME et al., 2017. Lactic acid bacteria and Bifidobacteria with potential to design natural biofunctional health-promoting dairy foods. Front Microbiol, 8: 846, doi: 10.3389/ fmicb.2017.00846

Lindberg JE, 2014. Fiber effects in nutrition and gut health in pigs. J Anim Sci Biotechnol, 5: 15, doi: 10.1186/2049-1891-5-15

Lo Verso L, Talbot G, Morissette B, Guay F, Matte JJ et al., 2020. The combination of nutraceuticals and functional feeds as additives modulates gut microbiota and blood markers associated with immune response and health in weanling piglets. J Anim Sci, 98(7): 1-16, doi: 10.1093/jas/ skaa208

Looft T, Allen HK, Cantarel BL, Levine U, Bayles DO et al., 2014. Bacteria, phages and pigs: the effects of in-feed antibiotics on the microbiome at different gut locations. ISME J, 8: 1566-1576, doi: 10.1038/ismej.2014.12

Mach N, Berri M, Estellé J, Levenez F, Lemonnier G et al., 2015. Early-life establishment of the swine gut microbiome and impact on host phenotypes. Environ Microbiol Rep, 7(3): 554-569, doi: $10.1111 / 1758-2229.12285$

McCormack UM, Curião T, Buzoianu SG, Prieto ML, Ryan T et al., 2017. Exploring a possible link between the intestinal microbiota and feed efficiency in pigs. Appl Environ Microbiol, 83(15): e00380-17, doi: 10.1128/AEM. 00380-17
McCormack UM, Curião T, Metzler-Zebeli BU, Wilkinson T, Reyer H et al., 2019. Improvement of feed efficiency in pigs through microbial modulation via fecal microbiota transplantation in sows and dietary supplementation of inulin in offspring. Appl Environ Microbiol, 85(22): 1-18, doi: 10.1128/AEM.01255-19

Montagne L, Pluske JR and Hampson DJ, 2003. A review of interactions between dietary fibre and the intestinal mucosa, and their consequences on digestive health in young non-ruminant animals. Anim Feed Sci Technol, 108(1-4): 95117, doi: 10.1016/S0377-8401(03)00163-9

Mu C, Bian G, Su Y and Zhu W, 2019. Differential effects of breed and nursing on early-life colonic microbiota and immune status as revealed in a cross-fostering piglet model. Appl Environ Microbiol, 85(9): e02510-18, doi: 10.1128/ AEM.02510-18

Owusu-Asiedu A, Patience JF, Laarveld B, Kessel AGV, Simmins PH et al., 2006. Effects of guar gum and cellulose on digesta passage rate, ileal microbial populations, energy and protein digestibility, and performance of grower pigs. J Anim Sci, 84(4): 843-852, doi: 10.2527/2006.844843x

Pajarillo EAB, Chae JP, Balolong MP, Kim HB, Seo KS et al., 2014. Pyrosequencing-based analysis of fecal microbial communities in three pure bred pig lines. J Microbiol, 52: 646-651, doi: 10.1007/s12275-014-4270-2

Pajarillo EAB, Chae JP, Balolong MP, Kim HB, Seo KS et al., 2015. Characterization of the fecal microbial communities of Duroc pigs using 16S rRNA gene pyrosequencing. Asian-Australas J Anim Sci, 28(4): 584-591, doi: 10.5713/ ajas.14.0651

Parkunan T, Das BC, Banerjee D, Mohanty N, Das PK et al., 2015. A comparative study on the expression profile of MCTs and HSPs in Ghungroo and Large White Yorkshire breeds of pigs during different seasons. Cell Stress Chaperones, 20(3): 441-449, doi: 10.1007/s12192-014-0569-5

Patil Y, Gooneratne R and Ju XH, 2020. Interactions between host and gut microbiota in domestic pigs: A review. Gut Microbes, 11(3): 310-334, doi: $10.1080 / 19490976.2019 .1690363$ 
Petri D, Hill JE and Van Kessel AG, 2010. Microbial succession in the gastrointestinal tract (GIT) of the preweaned pig. Livest Sci, 133(1-3): 107109, doi: 10.1016/j.livsci.2010.06.037

Pickard JM, Zeng MY, Caruso R and Núñez G, 2017. Gut microbiota: role in pathogen colonization, immune responses, and inflammatory disease. Immunol Rev, 279(1): 70-89, doi: 10.1111/imr.12567

Pieì S, Awati A, Vida S, Falluel I, Williams BA et al., 2007. Effects of added fermentable carbohydrates in the diet on intestinal proinflammatory cytokine-specific mRNA content in weaning piglets. J Anim Sci, 85(3): 673-683, doi: 10.2527/jas.2006-535

Pieper R, Vahjen W and Zentek J, 2015. Dietary fibre and crude protein: impact on gastrointestinal microbial fermentation characteristics and host response. Anim Prod Sci, 55(12): 1367-1375, doi: 10.1071/ AN15278

Rist VTS, Weiss E, Eklund M and Mosenthin R, 2013. Impact of dietary protein on microbiota composition and activity in the gastrointestinal tract of piglets in relation to gut health: A review. Animal, 7(7): 1067-1078, doi: 10.1017/ S1751731113000062

Schokker D, Zhang J, Zhang L, Vastenhouw SA, Heilig GHJ et al., 2014. Early-life environmental variation affects intestinal microbiota and immune development in new-born piglets. PLoS One, 9: e100040, doi: 10.1371/ journal.pone. 0100040

Shan T, Li L, Simmonds P, Wang C, Moeser A et al., 2011. The fecal virome of pigs on a high-density farm. J Virol, 85(22): 11697-11708, doi: 10.1128/JVI.05217-11

Shin D, Chang SY, Bogere P, Won K, Chai JY et al., 2019. Beneficial roles of probiotics on the modulation of gut microbiota and immune response in pigs. PLoS One, 14: 1-23, doi: 10.1371/journal.pone.0220843

Tan Z, Yang T, Wang Y, Xing K, Zhang F et al., 2017. Metagenomic analysis of cecal microbiome identified microbiota and functional capacities associated with feed efficiency in Landrace finishing pigs. Front Microbiol, 8: 1546, doi: 10.3389/fmicb.2017.01546
Tian L, Bruggeman G, van den Berg M, Borewicz K, Scheurink AJW et al., 2017. Effects of pectin on fermentation characteristics, carbohydrate utilization, and microbial community composition in the gastrointestinal tract of weaning pigs. Mol Nutr Food Res, 61(1): 186, doi: 10.1002/mnfr.201600186

Turner PV, 2018. The role of the gut microbiota on animal model reproducibility. Anim Model Exp Med, 1(2): 109-115, doi: 10.1002/ame2.12022

Umu ÖCO, Mydland LT, Øverland M, Press CM and Sørum H, 2020. Rapeseed-based diet modulates the imputed functions of gut microbiome in growing-finishing pigs. Sci Rep, 10: 1-12, doi: 10.1038/s41598-020-66364-4

Urubschurov V, Janczyk P, Souffrant W-B, Freyer G, Zeyner A et al., 2011. Establishment of intestinal microbiota with focus on yeasts of unweaned and weaned piglets kept under different farm conditions. FEMS Microbiol Ecol, 77(3): 493502, doi: 10.1111/j.1574-6941.2011.01129.x

Wang M and Donovan SM, 2015. Human microbiotaassociated swine: current progress and future opportunities. ILAR J, 56(1): 63-73, doi: 10.1093/ilar/ilv006

Wang M, Radlowski EC, Monaco MH, Fahey Jr. GC, Gaskins HR et al., 2013. Mode of delivery and early nutrition modulate microbial colonization and fermentation products in neonatal piglets. J Nutr, 143(6): 795-803, doi: 10.3945/ jn.112.173096

Wang T, Yao W, Li J, Shao Y, He Q et al., 2020. Dietary garcinol supplementation improves diarrhea and intestinal barrier function associated with its modulation of gut microbiota in weaned piglets. J Anim Sci Biotechnol, 11: 1-13, doi: 10.1186/ s40104-020-0426-6

Wang X, Tsai T, Deng F, Wei X, Chai J et al., 2019. Longitudinal investigation of the swine gut microbiome from birth to market reveals stage and growth performance associated bacteria. Microbiome, 7: 109, doi: 10.1186/s40168-0190721-7

Wei X, Tsai T, Knapp J, Bottoms K, Deng F et al., 2020. ZnO modulates swine gut microbiota and improves growth performance of nursery pigs 
when combined with peptide cocktail. Microorganisms, 8(2): 146, doi: 10.3390/ microorganisms 8020146

Wells JE, Yen JT and Miller DN, 2005. Impact of dried skim milk in production diets on Lactobacillus and pathogenic bacterial shedding in growingfinishing swine1. J Appl Microbiol, 99(2): 400407, doi: 10.1111/j.1365-2672.2005.02629.x

Yang H, Huang X, Fang S, He M, Zhao Y et al., 2017. Unraveling the fecal microbiota and metagenomic functional capacity associated with feed efficiency in pigs. Front Microbiol, 8: 1555, doi: 10.3389/fmicb.2017.01555

Yang H, Xiao Y, Wang J, Xiang Y, Gong Y et al., 2018a. Core gut microbiota in Jinhua pigs and its correlation with strain, farm and weaning age. J Microbiol, 56: 346-355, doi: 10.1007/s12275018-7486-8

Yang H, Yang M, Fang S, Huang X, He Met al., 2018b.
Evaluating the profound effect of gut microbiome on host appetite in pigs. BMC Microbiol, 18: 225, doi: $10.1186 / \mathrm{s} 12866-018-1364-8$

Yin Y-L, Tang ZR, Sun ZH, Liu ZQ, Li TJ et al., 2008. Effect of galacto-mannan-oligosaccharides or chitosan supplementation on cytoimmunity and humoral immunity in early-weaned piglets. AsianAustralas J Anim Sci, 21(5): 723-731, doi: 10.5713/ ajas.2008.70408

Zhang Y, Zhou J, Dong Z, Li G, Wang J et al., 2019. Effect of dietary copper on intestinal microbiota and antimicrobial resistance profiles of Escherichia coli in weaned piglets. Front Microbiol, 10: 2808, doi: 10.3389/ fmicb.2019.02808

Zhao W, Wang Y, Liu S, Huang J, Zhai Z et al., 2015. The dynamic distribution of porcine microbiota across different ages and gastrointestinal tract segments. PLoS One, 10: e0117441, doi: 10.1371/ journal.pone.0117441 